PROCEEDINGS OF THE

AMERICAN MATHEMATICAL SOCIETY

Volume 139, Number 7, July 2011, Pages 2553-2558

S 0002-9939(2010)10705-0

Article electronically published on December 30, 2010

\title{
CONCAVE UNIMODAL MAPS HAVE NO MAJORISATION RELATIONS BETWEEN THEIR ERGODIC MEASURES
}

JACOB STEEL

(Communicated by Bryna Kra)

\begin{abstract}
If $T$ is a concave unimodal map on the unit interval $[0,1]$ and $\left\{x: T^{n}(x)=1\right.$ for some $\left.n\right\}$ is dense in $[0,1]$, we prove that all $T$-invariant ergodic Borel probability measures are mutually incomparable with respect to the partial order of majorisation. This contrasts sharply with the situation for other interval maps previously studied.
\end{abstract}

The basic problem of ergodic optimisation is as follows: for a real-valued function $f: X \rightarrow \mathbb{R}$ on a space $X$ and a transformation $T: X \rightarrow X$, which $T$-invariant Borel probability measure $\mu$ gives the largest possible value of $\int_{X} f d \mu$ ? The case which has been most studied is when the set $X$ is the unit interval $I=[0,1]$, where results include the following. If $T$ is the doubling map $x \mapsto 2 x(\bmod 1)$ and $f$ is a function of the form $f(x)=\sin (2 \pi(x+\theta))$, then the unique maximising measure is one of a class of measures known as Sturmian (see [1 for the result and 22 for example for an introduction to Sturmian measures). If $f$ is a concave function, then its maximising measure is again Sturmian (see 6]), while if $T$ is the map $x \mapsto 2 x+\frac{1}{2}(\bmod 1)$ and $f$ is concave, then its maximising measure is the fixed point measure $\delta_{\frac{1}{2}}$ (see [10]). If $T$ is a piecewise continuous orientation-reversing expanding map such as the negative doubling map $T: x \mapsto-2 x(\bmod 1)$ and $f$ is concave, then the maximising and minimising measures are combinations of Dirac measures concentrated on fixed points (see [8]). For a treatment of ergodic optimisation in more general contexts, see for example [4].

An approach that has led to the identification of maximising measures for concave functions for various transformations of the unit interval relies on the following partial ordering of probability measures:

Definition 1. Let $\mu$ and $\nu$ be measures on $I$. Define $w_{\mu}(x):=\int_{0}^{x} \mu[0, s) d s$. We say that $\mu$ is majorised by $\nu$, written $\mu \prec \nu$, if $w_{\mu}(x) \leq w_{\nu}(x)$ for all $0 \leq x \leq 1$ and $w_{\mu}(1)=w_{\nu}(1)$.

Note that the barycentre of $\mu$, given by $\rho(\mu)=\int_{0}^{1} x d \mu(\mathrm{x})$, satisfies $\rho(\mu)=$ $1-w_{\mu}(1)$, so two measures have the same barycentre if and only if $w_{\mu}(1)=w_{\nu}(1)$. Heuristically, $\mu \prec \nu$ means that $\mu$ and $\nu$ have the same barycentre, but $\nu$ is more spread out than $\mu$.

The following is a well-known alternative formulation of majorisation:

Received by the editors March 18, 2010 and, in revised form, June 4, 2010 and July 12, 2010. 2010 Mathematics Subject Classification. Primary 37E05; Secondary 37C40. 
Lemma 1. If $\mu \prec \nu$, then $\int_{0}^{1} f(x) d \mu(x) \leq \int_{0}^{1} f(x) d \nu(x)$ for convex $f$ and $\int_{0}^{1} f(x) d \mu(x) \geq \int_{0}^{1} f(x) d \nu(x)$ for concave $f$.

This lemma is proved by noting, via integration by parts, that $\int_{0}^{1} f d m=$ $\int_{0}^{1}\left(\int_{0}^{x} m[0, s) d s\right) f^{\prime \prime}(x) d x$ for any signed measure $m(=\mu-\nu)$ satisfying $m[0,1]=0$ and $\int_{0}^{1} x d m(x)=0$. A consequence of Lemma 1 is the following: if a transformation $T: I \rightarrow I$ is such that for each $\rho \in[0,1]$ there is a $T$-invariant Borel invariant probability measure $\mu_{\rho}$ of barycentre $\rho$ which majorises all other such measures of barycentre $\rho$, then for any concave function $f$, the maximising $T$-invariant probability measure must be one of the $\mu_{\rho}$, for some value $\rho$.

This approach was exploited in [5, 6] in the context of the doubling map $T(x)=$ $2 x(\bmod 1)$, one of the standard examples of a chaotic dynamical system. An attractive feature of this map is that different periodic orbits with a common barycentre are readily exhibited: it suffices to choose orbits of the same period whose symbolic representations (with respect to the usual dyadic coding) have the same number of 1's. Majorisation relations between (the invariant measures supported by) these periodic orbits can then be computed directly and are seen to be rather non-trivial (see [5, 6, 7]).

By contrast for the tent map $T(x)=1-|2 x-1|$, another favourite textbook example of a chaotic dynamical system, it is much harder to find periodic orbits with the same barycentre. After the initial observation by Misiurewicz [9] that such orbits do exist, Chen and Dong [3] provided further insight, in particular proving that arbitrarily many periodic orbits may share a common barycentre. This suggests the problem of investigating possible majorisation relations between such periodic orbits. However it turns out that, with respect to the majorisation partial order, none of the periodic orbits for the tent map are comparable to each other. This surprising fact is a consequence of the following result, which applies more generally to ergodic invariant measures for certain concave unimodal maps:

Theorem 1. Let $T: I \rightarrow I$ be unimodal (i.e. there exists $a \in I$ such that $\left.T\right|_{[0, a]}$ is increasing and $\left.T\right|_{[a, 1]}$ is decreasing), concave, and such that $\left\{x: T^{n}(x)=\right.$ $T(a)$ for some $n\}$ is dense in $I$.

If $\mu, \nu$ are $T$-invariant ergodic measures on $I$, with $\mu \prec \nu$, then necessarily $\mu=\nu$; in other words, no non-trivial majorisation can occur among such measures.

Remark 1. In terms of ergodic optimisation, a consequence of Theorem 1 is that, by contrast to the situation for the doubling map (cf. [5, 6, 7]), for concave unimodal $T$ with $\left\{x: T^{n}(x)=T(a)\right.$ for some $\left.n\right\}$ dense, no class of invariant measures plays a privileged role in terms of maximising the integrals of concave functions.

Proof of Theorem 1. Let $A:=\left\{x: T^{n}(x)=T(a)\right.$ for some $\left.n\right\}$ denote the set of iterated preimages of the maximum. Let $\mu, \nu$ be $T$-invariant ergodic Borel probability measures with $\mu \prec \nu$. We will show that $\mu \equiv \nu$ on the closure of $A$. Since $T$ has a maximum at $a, \mu((T(a), 1])=0$. So, by rescaling, without loss of generality we may assume that $T(a)=1$. Similarly, if $T(1)>0$, then $\mu(0, T(1))=0$, and the Dirac measure at the leftmost (fixed) point 0 is clearly incomparable to all other measures (none of which share its barycentre), so without loss of generality we may assume that $T(1)=0$.

Let $T_{-}^{-1}(x)$ and $T_{+}^{-1}(x)$ be the left-half and right-half preimages of $x$, respectively, or $T_{-}^{-1}(x)=0$ if $x<T(0)$. By the above assumption, these are well-defined. 
Next, we focus attention on two of the $2^{n}$ possible $n$th bounds of preimages of a point $x$ (if $T(0)=0$, these will all be $n^{\text {th }}$ order preimages of $x$; if not, then at least some of them will be) defined by

$$
T_{--}^{-n}(x):=\left(T_{-}^{-1}\right)^{n}(x) \quad \text { and } \quad T_{+-}^{-n}(x):=T_{+}^{-1}\left(T_{-}^{-(n-1)}(x)\right) .
$$

In particular, note that if $y>T_{+-}^{-n}(1)$, then $T_{+-}^{-n}\left(T^{n}(y)\right)=T^{n}\left(T_{+-}^{-n}(y)\right)=y$. This is only possible if $T_{+-}^{-n}(1)<1$.

We now consider constraints imposed on $w_{\mu}$ by the $T$-invariance of $\mu$. By definition $w_{\mu}(x)=\int_{0}^{x} \mu[0, s) d s$, and

$$
\begin{aligned}
\mu[0, s) & =\mu\left[0, T_{-}^{-1}(s)\right)+\mu\left(T_{+}^{-1}(s), 1\right] \\
& =\mu\left[0, T_{-}^{-2}(s)\right)+\mu\left(T_{+-}^{-2}(s), 1\right]+\mu\left(T_{+-}^{-1}(s), 1\right] \\
& =\lim _{n \rightarrow \infty} \mu\left[0, T_{--}^{-n}(s)\right)+\sum_{n=1}^{\infty} \mu\left(T_{+-}^{-n}(s), 1\right] .
\end{aligned}
$$

Clearly the Dirac measure concentrated at the fixed point 0 does not majorise any other measure, and since we are only interested in majorisation among ergodic measures $\mu$, we may assume that $0=\mu(\{0\})$. Therefore $0=\lim _{n \rightarrow \infty} \mu\left[0, T_{--}^{-n}(s)\right)$ for any $s$, so

$$
\mu[0, s)=\sum_{n=1}^{\infty} \mu\left(T_{+-}^{-n}(s), 1\right] .
$$

We are interested in the difference between $w_{\mu}$ and $w_{\nu}$. Defining $\delta$ to be the signed measure $\delta=\mu-\nu$ and $w_{\delta}=w_{\mu}-w_{\nu}$, we see that

$$
w_{\delta}(x)=\int_{0}^{x} \sum_{n=1}^{\infty} \delta\left(T_{+-}^{-n}(s), 1\right] d s=\sum_{n=1}^{\infty} \int_{0}^{x} \delta\left(T_{+-}^{-n}(s), 1\right] d s
$$

since $\sum_{n=1}^{k} \nu\left[0, T_{+-}^{-n}(s)\right]-\mu\left[0, T_{+-}^{-n}(s)\right]$ is a monotone function of $s$ for sufficiently large $k$. Note also that $\delta[0,1]=0$, as $\delta$ is the difference of two probability measures.

Now $T$ need not be smooth, but since it is concave, the first derivative is defined except at countably many points, the left and right derivatives are defined everywhere and the second derivative can be defined everywhere as a sum of a real-valued function and a countable number of Dirac delta functions. The same is true of $T^{n}$.

Suppose that $x>T_{+-}^{-n}(1)$. It follows that $x>a$ and $T^{i}(x)<a$ for $1 \leq i \leq n-1$. Unimodality of $T$ then implies that $T^{\prime}(x)<0$ and $T^{\prime}\left(T^{i} x\right)>0$ for $1 \leq i \leq n-1$, and hence

$$
\left(T^{n}\right)^{\prime}(x)=\prod_{i=0}^{n-1} T^{\prime}\left(T^{i-1}(x)\right)<0 \quad \text { for } x \in\left[T_{+-}^{-n}(1), 1\right] .
$$

In addition, concavity of $T$ means that $T^{\prime \prime} \geq 0$, so

$$
\left(T^{n}\right)^{\prime \prime}(x)=\sum_{i=0}^{n-1} T^{\prime \prime}\left(T^{i} x\right) \prod_{j=i+1}^{n-1} T^{\prime}\left(T^{j} x\right)\left(\prod_{j=0}^{i-1} T^{\prime}\left(T^{j} x\right)\right)^{2} \leq 0 \text { for } x \in\left(T_{+-}^{-n}(1), 1\right] .
$$

Now

$$
w_{\delta}(x)=\sum_{n=1}^{\infty} \int_{0}^{x} \delta\left(T_{+-}^{-n}(s), 1\right] d s=\sum_{n=1}^{\infty} \int_{0}^{x}-\delta\left[0, T_{+-}^{-n}(s)\right] d s .
$$


Letting $y=T_{+-}^{-n}(s)$ and noting that $T_{+-}^{-n}(x) \geq T_{+-}^{-n}(1)$ implies that either $T_{+-}^{-n}(x)$ $=1$ or $T^{n}(y)=s$, we obtain

$$
w_{\delta}(x)=\sum_{n=1}^{\infty} \int_{T_{+-}^{-n}(x)}^{1} \delta[0, y]\left(T^{n}\right)^{\prime}(y) d y=\sum_{n=1}^{\infty} \int_{T_{+-}^{-n}(x)}^{1} w_{\delta}^{\prime}(y)\left(T^{n}\right)^{\prime}(y) d y .
$$

Integration by parts then gives

$$
\begin{aligned}
w_{\delta}(x)=\sum_{n=1}^{\infty}\left(w_{\delta}(1)\left(T^{n}\right)^{\prime}(1)-\right. & w_{\delta}\left(T_{+-}^{-n}(x)\right)\left(T^{n}\right)^{\prime}\left(T_{+-}^{-n}(x)\right) \\
& \left.-\int_{T_{+-}^{-n}(x)}^{1} w_{\delta}(y)\left(T^{n}\right)^{\prime \prime}(y) d y\right) .
\end{aligned}
$$

Now suppose that $\mu$ majorises $\nu$. It follows that $w_{\delta}(0)=w_{\delta}(1)=0$ and $w_{\delta} \geq 0$. Setting $x=1$ in (3) gives

$$
0=w_{\delta}(1)=\sum_{n=1}^{\infty}\left(-w_{\delta}\left(T_{+-}^{-n}(1)\right)\left(T^{n}\right)^{\prime}\left(T_{+-}^{-n}(1)\right)-\int_{T_{+-}^{-n}(1)}^{1} w_{\delta}(y)\left(T^{n}\right)^{\prime \prime}(y) d y\right),
$$

and hence

$$
\sum_{n=1}^{\infty} w_{\delta}\left(T_{+-}^{-n}(1)\right)\left(T^{n}\right)^{\prime}\left(T_{+-}^{-n}(1)\right)=-\sum_{n=1}^{\infty} \int_{T_{+-}^{-n}(1)}^{1} w_{\delta}(y)\left(T^{n}\right)^{\prime \prime}(y) d y \geq 0
$$

because $\left(T^{n}\right)^{\prime \prime} \leq 0$ on $\left[T_{+-}^{-n}(1), 1\right]$, by (2) .

Now $w_{\delta}$ is non-negative and not identically zero (since $\mu \neq \nu$ ), and (11) gives $\left(T^{n}\right)^{\prime}\left(T_{+-}^{-n}(1)\right) \leq 0$ for each $n \geq 1$, so the only way that (4) can be satisfied is if

$$
w_{\delta}\left(T_{+-}^{-n}(1)\right)=0 \text { for all } n \geq 1 \text {. }
$$

We can now apply the above argument iteratively. For any $m \geq 1$, set $x=$ $T_{+-}^{-m}(1)$ in (3), and using (15) (with $n=m$ ), we see that

$$
\begin{aligned}
0 & =w_{\delta}\left(T_{+-}^{-m}(1)\right) \\
& =\sum_{n=1}^{\infty}\left(-w_{\delta}\left(T_{+-}^{-n} T_{+-}^{-m}(1)\right)\left(T^{n}\right)^{\prime}\left(T_{+-}^{-n} T_{+-}^{-m}(1)\right)-\int_{T_{+-}^{-n} T_{+-}^{-m}(1)}^{1} w_{\delta}(y)\left(T^{n}\right)^{\prime \prime}(y) d y\right),
\end{aligned}
$$

and hence

(6)

$$
\sum_{n=1}^{\infty} w_{\delta}\left(T_{+-}^{-n} T_{+-}^{-m}(1)\right)\left(T^{n}\right)^{\prime}\left(T_{+-}^{-n} T_{+-}^{-m}(1)\right)=-\sum_{n=1}^{\infty} \int_{T_{+-}^{-n} T_{+-}^{-m}(1)}^{1} w_{\delta}(y)\left(T^{n}\right)^{\prime \prime}(y) d y \geq 0,
$$

and by arguing as above we deduce that necessarily

$$
w_{\delta}\left(T_{+-}^{-n} T_{+-}^{-m}(1)\right)=0 \text { for all } m, n \geq 1 .
$$

By repeating this process we see that $w_{\delta}(x)=0$ whenever $x$ is an iterated preimage of 1 . Since the set of these points is dense by assumption and $w_{\delta}$ is continuous, this implies that $w_{\delta}$ is zero everywhere, and so the measures $\mu$ and $\nu$ must be identical, completing the proof.

From the above proof it is clear that even if the set $A=\left\{x: T^{n}(x)=T(a)\right.$ for some $n\}$ is not dense, if one $T$-invariant ergodic measure majorises another, then the function $w_{\delta}$ defined above must be identically zero on the closure of $A$, as illustrated by the following examples. 
Examples. Consider the map $T_{a}: I \rightarrow I$ defined by $T_{a}(x)=\frac{x}{2}+\frac{3}{4}$ for $0 \leq x \leq \frac{1}{2}$ and $T_{a}(x)=2-2 x$ for $\frac{1}{2} \leq x \leq 1$. Here points in the open intervals $\left(0, \frac{1}{2}\right)$ and $\left(\frac{3}{4}, 1\right)$ are never iterated onto 1 , so these intervals do not contain any preimages of 1 ; thus the density hypothesis of Theorem 1 is not satisfied. Note that the (ergodic) $T_{a}$-invariant measure supported on the period- 4 orbit $\left\{0, \frac{1}{2}, \frac{3}{4}, 1\right\}$ does majorise the (ergodic) $T_{a}$-invariant measure supported on the period-2 orbit $\left\{\frac{1}{4}, \frac{7}{8}\right\}$. However, for these two measures the function $w_{\delta_{a}}$ (as defined in the proof of Theorem 1) is zero except on the open intervals $\left(0, \frac{1}{2}\right)$ and $\left(\frac{3}{4}, 1\right)$, which contain no preimages of 1 .

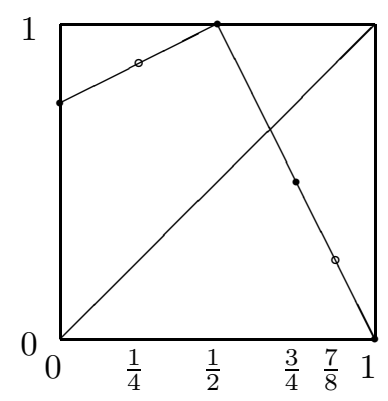

$T_{a}$

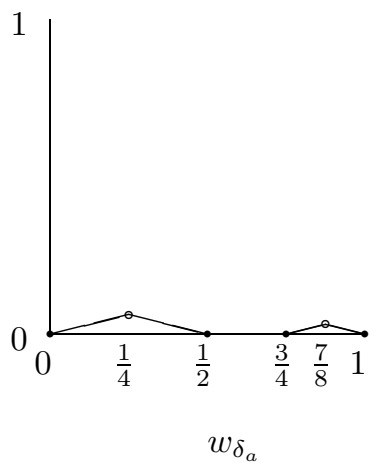

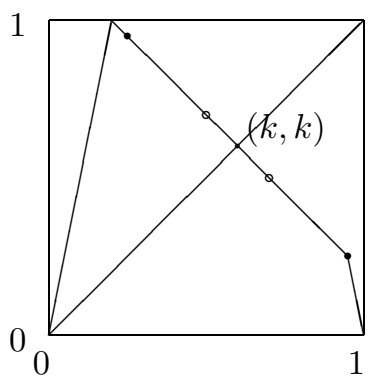

$T_{b}$

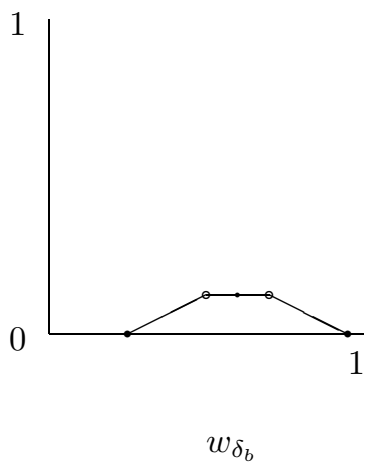

Alternatively, consider any map $T_{b}: I \rightarrow I$ such that $T_{b}(x)=k-x$ for some interval containing the point $k$, where $\frac{1}{2}<k<1$. Such a map has infinitely many period-2 orbits (any pair of points of the form $\left(\frac{k}{2}+\epsilon, \frac{k}{2}-\epsilon\right)$ is a period-2 orbit), whose corresponding (ergodic) invariant measures majorise each other, as well as the Dirac measure concentrated on the fixed point $k / 2$. Again, note that for such pairs of measures the function $w_{\delta_{b}}$ will be 0 except on the interval of periodic points.

\section{ACKNOWLEDGEMENTS}

This work was supported by an EPSRC PhD studentship. The author is grateful to Henk Bruin and Oliver Jenkinson for their comments on this work. 


\section{REFERENCES}

[1] T. Bousch, Le poisson n'a pas d'arêtes, Ann. Inst. Henri Poincaré (Proba. et Stat.), 36 (2000), 489-508. MR.1785392(2001i:37005)

[2] S. Bullett and P. Sentenac, Ordered orbits of the shift, square roots and the devil's staircase, Math. Proc. Camb. Phil. Soc., 115 (1994), 451-481. MR:1269932 (95j:58043)

[3] K.-C. Chen and X. Dong, On the barycenter of the tent map, Proc. Amer. Math. Soc., 138 (2010), 4025-4035.

[4] O. Jenkinson, Ergodic Optimization, Discr. \& Cont. Dyn. Sys., 15 (2006), 197-224. MR2191393 (2009i:37075)

[5] O. Jenkinson, Optimization and majorization of invariant measures, Elec. Res. Ann. Amer. Math. Soc., 13 (2007), 1-12. MR2285761(2007m:37006)

[6] O. Jenkinson, A partial order on $\times 2$-invariant measures, Math. Res. Lett., 15 (2008), 893-900. MR2443990 (2010h:37005)

[7] O. Jenkinson, Balanced words and majorization, Discr. Math. Alg. $\mathscr{G}$ Appl., 1 (2009), 485498.

[8] O. Jenkinson and J. Steel, Majorization of invariant measures for orientation-reversing maps, Ergod. Th. \& Dyn. Sys., 30 (2010), 1471-1483.

[9] M. Misiurewicz, Rotation theory, Proceedings RIMS Workshop on Dynamical Systems and Applications, 2006 (http://www.math.kyoto-u.ac.jp/ kokubu/RIMS2006/proc.html).

[10] J. Steel, Barycentres and majorisation for invariant measures of the shifted doubling map, preprint, 2010.

School of Mathematics, Queen Mary University of London, Mile End Road, London E1 4NS, United Kingdom 